\title{
Distributed Adaptive Design with Hierarchical Autonomous Graph Transformation Systems
}

\author{
Leszek Kotulski ${ }^{1}$ and Barbara Strug ${ }^{2}$ \\ ${ }^{1}$ Department of Automatics, AGH University of Science and Technology \\ Al. Mickiewicza 30, 30059 Krakow, Poland \\ ${ }^{2}$ Department of Physics, Astronomy and Applied Computer Science, \\ Jagiellonian University, Reymonta 4, Krakow, Poland \\ kotulski@agh.edu.pl, uistrug@if.uj.edu.pl
}

\begin{abstract}
In this paper a graph transformation system with the parallel derivation is used to model the process of distribution and adaptation for computer aided design. It is based on earlier research in formal language theory, especially graph grammars, and distributed models. The motivation for the ideas presented here is given and possible ways of application are described. A connection to the multi-agent model is also presented.
\end{abstract}

Keywords: Graph transformations, grammar systems, design.

\section{Introduction}

Distributed model of computing is becoming more and more popular, especially with a rapid development of the Internet and availability of distributed development platforms. Such a model seems to be very useful in the domain of computeraided design. Many design problems can be divided into a number of tasks, each of them carried out by an autonomous agent either only occasionally exchanging pieces of information or contributing its capabilities to a common object being designed. Yet no formal model of such a cooperative distributed design has been proposed so far.

Real world objects being designed are usually complex and contain a number of different sub-elements inter-related in different ways. To represent such complex objects in different domains of computer science graphs are very often used [15. Their ability to represent the structure of an object as well as the different types of relations between its parts makes them particularly useful in computer aided design. The process of designing objects requires thus a method of generating graphs .

A new approach to graph generation in computer aided design domain, proposed in this paper, uses cooperation and distribution as a basis for generating designs with the application of graph grammars. It is based on earlier research in the domain of application of the theory of formal languages to the computer aided design 667] and distributed model of computation [5|15/11. In particular graph grammars [16/8,13] and grammar systems [2]34] were used as the inspiration for this research. 


\section{Graph Structures in the Design Process}

One of the most useful representations in computer aided design systems is based on graphs. The process of designing can then be seen as generating graphs representing some objects. In such situation introducing a method for the generation of these graphs is needed. Moreover such a method must guarantee that graphs it produces are valid in respect to a design problem being solved. In other words each graph generated by a chosen method must be interpretable as a design. Usually it must also satisfy some design criteria or constraints. Graph transformations are a method fulfilling all above requirements.

In real world problems the object being designed are complex and consist of many parts which in turn can contain subparts. Thus the graphs representing such an object can become large structures difficult to maintain, update and analyze. Yet in many situations design decisions dealing with one part of the design are independent from the rest of it. Thus it seems reasonable to distribute the graph representing such a design into a number of local graphs, each representing a part of design, and a master graph containing the overall structure of the design. Each local graph can be generated by its own graph transformation system Graph transformation system can thus be considered a set of autonomous systems paralelly cooperating with the user (designer) thus behaving itself like an agent.

Using such an approach, two major problems inherent to graph-based representations in design problems, namely the size of graphs and number of transformation rules, can be reduced.

Moreover there is nothing to prevent local graph representing a part of a design to be a master one either for other graphs representing subparts of this part (what introduce hierarchy inside graph) or the common (replicated) part of the graph structure. The second one will be discussed in the paper in a more detailed way.

Distribution of the representation solves mentioned problems but also generates new ones. As each graph represents a part of a design then there can exist elements that can be represented in different subgraphs. Thus a need for synchronization any modification of their environment arises. For example in a house design a system expected to design a floor layout of a house with the whole infrastructure and furnishing each room can be considered a valid part of a design and the master graph would contain the structure of the house layout and common elements. The way a furniture is placed in one room is independent from whatever happens to be placed in other rooms, thus each one can be designed by an autonomous agent. Yet some common elements exist. Doors can be one of such elements as they are shared by two rooms and thus changing their position must be synchronized inside both graphs representing these rooms, thus a need for communication between agents arises.

Moreover there are some limited resources to be placed in the house. For example it may be specified that two TV sets must be placed in the house with 
some additional constraints like: the distance from TV to a seat is at least 2 meters but no more then five. Thus the master unit broadcasts the resource and waits for responses from all rooms. Each local agent responds by specifying a measure of satisfying the constraints and the master unit decides in which room the TV would be placed, but the exact position is decided by the local system (agent). Let us, however, note that the centralization of some data and decisions inside master unit can create bottlenecks effect and decrease the effectiveness of the system, so we will sketch the possibility of the direct cooperation of the distributed components. The decision on both the distribution of the design data structures and the decomposition of the design process generates a need to find a method of synchronizing and negotiating the work on replicated description of elements and placement of limited resources.

In the paper we use for this purpose the concept of the Derivation Control Environment, presented in the next chapter, that controls the graph transformation with the help of productions of the grammar associated with it.

\section{Derivation Control Environment}

The main objectives of introducing the Derivation Control Environment (DCE) are:

- the support for local derivation of the graph,

- the representation of users reactions,

- the synchronization of both user's reaction with the proper action on the local graph and the derivation of the local cooperation.

Thus DCE consist of two types of units: - the local Derivation Control Diagram (DCD), - local Users Environments (UE). The definition of the communication mechanism is one of the most important properties of the distributed environment. We assume that both types of units are able to serve messages in the form of requests that have a unique name, and carry the values of attributes associated with this type of request (see [1] for formal definition).

We demand from the communication environment that the sent request be delivered to the destination unit in a finite time. We assume that each request has at least two attributes defined: sender and time_stamp defining appropriately the unit and the time of the generated request. Let RS denote the set of all possible requests, so any finite subset of $\mathrm{RS}$ will be called a requests set.

We assume that UE is characterized only by the ability of generating (internal) requests of services to DCD and servicing (external) requests required by DCDs. DCD unit is responsible both for modifying the maintained local graph as reaction to the appearance of some requests and generating sequence of requests to other units in order to synchronize its action with other units. So, it should introduce: 
1. a notion for describing some constrains in applying the grammar production in a given context (as analogy to the OCL correlated with algebraic transformation [5]);

2. the ability to synchronize, at the level of the single production, in order to wait until some specified sequence of events appears;

3. the ability to designate some sequence of productions associated with the given event.

Let us start to formalize these demands.

Definition 1. A derivation control diagram is a sixtuple $S=(N, I, F, T, \Pi$, Wait), where:

- $N$ - is the set of control points,

$-I \subset N$ and $F \subset N$ are accordingly, the set of starting control points and the set of final control points,

- $T$ is a set of transitions of the form $(k, q, P, S F)$, where:

- $k, q$ are control points,

- $P$ is either a production or $\emptyset$ symbol when no production is associated with this transition,

- SF is a semantic action described,

$-\Pi=\left\{\Pi_{k}\right\}$, it the set of selectors, ,

- Wait $=\left\{\right.$ Wait $\left._{k}\right\}$, is the set of synchronizing functions.

The synchronizing function $W a t_{k}$ awaits evaluation of the selector $\Pi_{k}$ until the condition it specified is fulfilled. Selector $\Pi_{k}$ points: a production, a semantics action and a destination control point associated with the chosen transition. Initially activity is associated with each starting point; when the transition is fired the activity is moved to the destination one. If the source control point is a starting one then the activity is also replicated to it; if the destination node is the final one the activity is cancelled. We assume that for any $\mathrm{k}$ the successful evaluation of the synchronizing function Wait $k_{k}$ and of the selector $\Pi_{k}$ is made in the critical section over the graph $\mathrm{G}$ and the set of request $\omega$. These assumptions imply not only the correctness of the sequential evaluation of the conditions defined inside control point, but also exclude busy form of waiting during evaluation of the awaiting synchronizing conditions. Like in Ada language, in the worst case all the synchronizing conditions are evaluated after any modification $\mathrm{G}$ or $\omega$. If no transition are fired to modify $\mathrm{G}$ or $\omega$ the evaluation is suspended until some new request $\mathrm{r}$ appears $\left.\left(\omega^{\prime}=\{r\} \cup \omega\right)\right)$. The semantic function SF (associated with the transition)

- enriches external request set (requesting some actions either of the designing system or the user),

- removes the request, that is serviced, from $\omega$,

- evaluate parameters of the right-hand graph of the production P. Production $\mathrm{P}$ is applied to current graph $\mathrm{G}$ and new graph $\mathrm{H}$ is created 
More intuitively, DCD can be interpreted as a graph connecting control points (see fig. 1) inside of which both the synchronizing function and selector, choosing one of the transitions from one control point to another one (drawn as an edge), are sequentially evaluated. During such a transition both production $P_{i}$ is applied and semantic action $S F_{i}$ is executed.

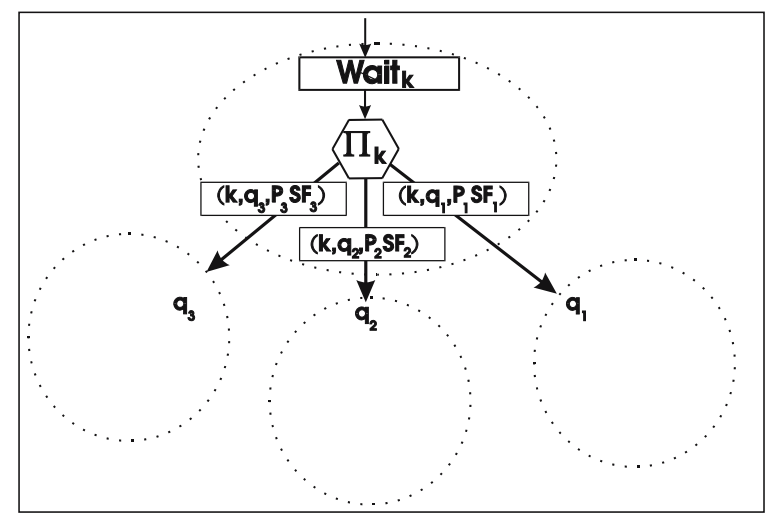

Fig. 1. Illustration of DCD

The Derivation Control Environment (DCE) is responsible for controlling both the local graphs modification and the whole distributed system cooperation.

Let us note that a local graph will be modified when a new request appears initially from some UE (representing user decision). Next requests can be generated as an effect of semantic actions executed by DCD during the service of these actions in order to make cooperation between DCDs . There are two particular reasons for the coordination of local activities:

- firstly some graph nodes can be replicated in several local graphs (describing the same element), so any action modifying its state (e.g. adding or removing any edge) needs cooperation of all these graph environments;

- secondly one can need some information about a global state of the system, so it has to ask the other DCDs to help in finding the global answer.

In the next section, these problems will be discussed in the context of adaptation in design.

\section{Synchronization and Adaptation in Design}

In the approach presented in this paper, we use labelled and attributed graphs to represent structures of design objects as they allow us to express multi-argument relations between objects' parts. Attributing is used to represent features of 


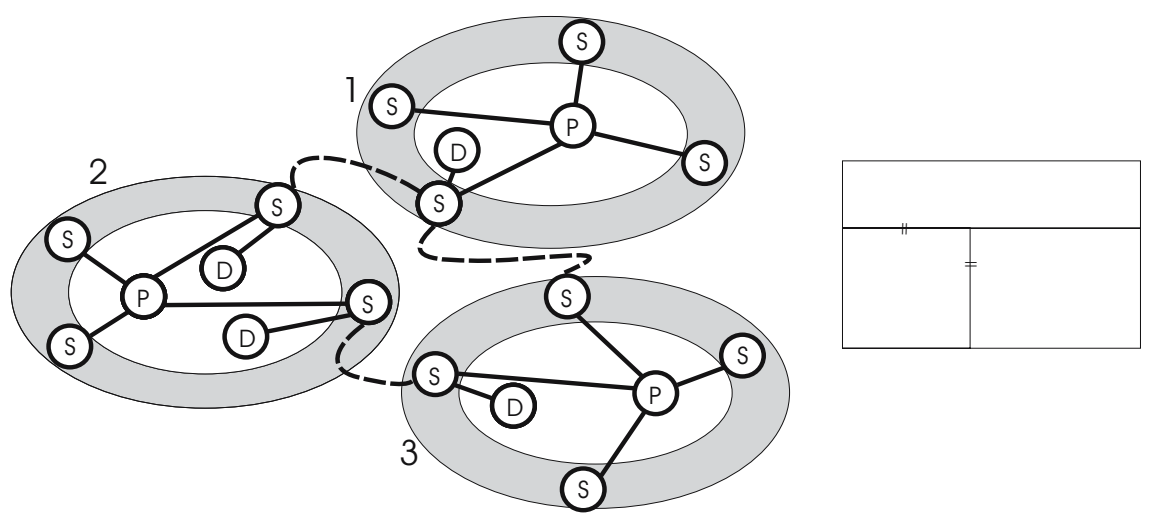

Fig. 2. Example representing a house layout

objects and relations between them. Attributes represent properties (for example size, position, colour or material) of elements corresponding to a given node or edge.

The graphs representing designs may be dynamically generated by means of so called graph grammars [15. Intuitively, such a grammar is a system of graph transformations, which consist of formal rules called productions. Each production is composed of a left-hand side. The left-hand side is replaced by the right-hand one only if it appears in the graph to be transformed. In each step a production is applied to a current graph. The sequence of such replacements, which starts from the initial graph, is called a derivation process.

Yet in real world design problems it is difficult, if not impossible, to define such an order of application a priori.

Designing is an incremental process of applying changes, evaluating and correcting designs. Moreover it must adapt to changes in design requirements that are not always known before the process starts. Note that it is very important that DCD is able to designate the derivation process by responding to events rather then following a predefined route. Each event generates one or more requests sent to other DCDs. For example adding a fireplace to a single room may require moving doors, which belong to two different rooms and thus appropriate requests must be generated; both DCDs (controlling graph representing these rooms) must react to them. Let consider this example in detail. Figure 2 depicts a graph representing a part of a house layout during a design process. It consist of three local graphs, representing three rooms. The replicated (shared) elements are placed in gray part of each graph and connected by dashed lines (it means that a connection is established by appropriate attribute(s) used in the request) Now a designer wants to add a fireplace to a room 2. Such an operation is local to the room 2 and is done by applying an appropriate production locally. Local application of productions has been widely and thoroughly presented in other papers [6] so it will be skipped here. While doing it a designer finds that the best location for the fireplace collides with the doors. So a decision is taken to remove the doors. A production Pr is applied that performs this action (shown 


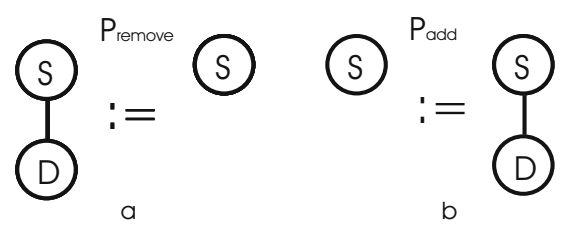

Fig. 3. Productions for adding and removing doors

in fig. 3a). But the doors are shared by the "room 2" with the "room 3". So the request remove_door (...) must be sent to DCD controlling the graph describing the room 3. This DCD should react to the appearance of the remove_door(...) request by applying the production Pr to remove the door from the "room 3". In both cases the removed doors can be the only door in the room. Checking the existence of the correct communication from the room can be made in our model with help of semantic action. If the communication stays correct than current thread of control can be moved to the starting point, otherwise it is moved to the part of DCD's responsible for negotiation adding different doors in other walls. Such doors are added by applying production shown in fig. 3 b.

\section{Conclusions}

In this paper we have shown that it is possible to decompose a complex design problem into a number of tasks. These tasks can be performed independently by agents working according to local grammars and cooperating when a need for updating shared resources arises. In the presented simple example all three graphs are derived with the use of the same grammar (thus all agents following the same rules) but there is no such requirement in the theoretical model we propose. Thus each graph may be derived by a different grammar. Moreover the grammars may belong to different class. The only requirement is that each grammar contains productions for updating shared elements.

In papers [11/12 it was shown that such an approach can be realized as a multi-agent system. Each graph transformation system can be considered to be an agent whose set of possible actions depends on a set of productions of the given GTS.

\section{References}

1. Borkowski A., Grabska E., Nikodem P, and Strug B. Searching for Innovative Structural Layouts by Means of Graph Grammars and Esvolutionary Optimization, Proc. 2nd Int. Structural Eng. And Constr. Conf, Rome (2003).

2. E. Csuhaj-Varjú, J. Dassow, J. Kelemen and Gh. Paun. Grammar systems. A grammatical approach to distribution and cooperation. Topics in Computer Mathematics 8. Gordon and Breach Science Publishers, Yverdon, 1994.

3. E. Csuhaj-Varjú: Grammar systems: A short survey, Proceedings of Grammar Systems Week 2004, 141-157, Budapest, Hungary, July 5-9, 2004. 
4. J. Dassow, Gh. Paun, and G. Rozenberg. Grammar systems. In A. Salomaa and G. Rozenberg, editors, Handbook of Formal Languages, volume 2, chapter 4, pp. 155-213, Springer-Verlag, Berlin-Heidelberg, 1997

5. Ehrig, H and Taentzer. G, Graphical represenation and graph transformations. ACM Comput. Surv., 31(3):9, 1999.

6. E.Grabska. Theoretical Concepts of Graphical Modelling. Part one: Realization of CP-graphs. Machine GRAPHICS and VISION, 2(1), pp. 3-38, 1993.

7. E. Grabska. Graphs and designing. Lecture Notes in Computer Science, 776 (1994).

8. E. Grabska, P. Nikodem, B. Strug. Evolutionary Methods and Graph Grammars in Design and Optimization of Skeletal Structures Weimar, 11th International Workshop on Intelligent Computing in Engineering, Weimar, 2004.

9. E. Grabska, B. Strug, Applying Cooperating Distributed Graph Grammars in Computer Aided Design, Parallel Processing and Applied Mathematics PPAM 2005, Lecture Notes in Computer Science, Springer 2006

10. E. Grabska, K. Grzesiak-Kopeć, J. Lembas, A. Łachwa and G. Ślusarczyk, Hypergraphs in design, Computational Imaging and Vision, Kluwer (to appear)

11. Kotulski, L. A Model of Software Genaration in a Distributted Environment by Graph Grammars, (in Polish) Habilitation Thesis,. Jagiellonian University Publishing, ISBN 83-233-1391-1, Krakow, 2000

12. Kotulski, L,Supporting Software Agents by the Graph Transformation Systems. Proc. of International Conference on Computational Science , LNCS 3993, pp887890, Springer 2006

13. P. Nikodem and B. Strug. Graph Transformations in Evolutionary Design, ICAISC 2004, Lecture Notes in Computer Science,vol 3070, pp. 456-461, Springer, 2004.

14. Gh. Paun and A. Salomaa editors, Grammatical models of multi-agent systems. Gordon and Breach, Amsterdam, 1999.

15. Rozenberg, G. Handbook of Graph Grammars and Computing by Graph. Transformations, vol.1 Fundations, World Scientific London (1997). 